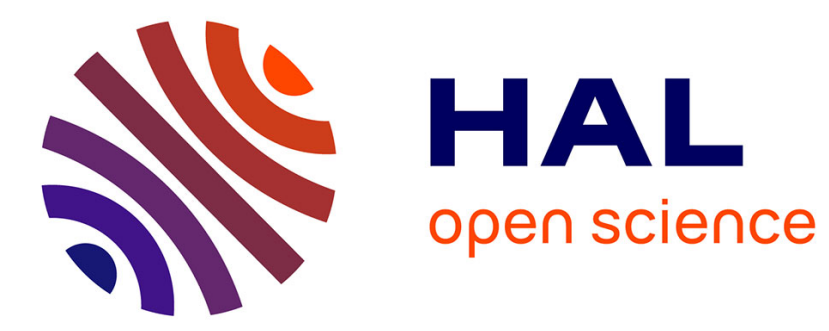

\title{
Mieux manager pour mieux soigner
}

Philippe Colombat, Julien Lejeune, Antonia Altmeyer, Evelyne Fouquereau

\section{To cite this version:}

Philippe Colombat, Julien Lejeune, Antonia Altmeyer, Evelyne Fouquereau. Mieux manager pour mieux soigner. Bulletin du Cancer, 2019, 106 (1), pp.55-63. 10.1016/j.bulcan.2018.10.012 . hal03183795

\section{HAL Id: hal-03183795 \\ https://hal.science/hal-03183795}

Submitted on 22 Oct 2021

HAL is a multi-disciplinary open access archive for the deposit and dissemination of scientific research documents, whether they are published or not. The documents may come from teaching and research institutions in France or abroad, or from public or private research centers.
L'archive ouverte pluridisciplinaire HAL, est destinée au dépôt et à la diffusion de documents scientifiques de niveau recherche, publiés ou non, émanant des établissements d'enseignement et de recherche français ou étrangers, des laboratoires publics ou privés.

\section{다)(1) $\$$}

Distributed under a Creative Commons Attribution - NonCommerciall 4.0 International 


\section{Mieux manager pour mieux soigner}

\section{A better management for a better care}

\section{Philippe COLOMBAT ${ }^{1 *}$, Julien LEJEUNE ${ }^{2}$, Antonia ALTMEYER ${ }^{3}$, Evelyne FOUQUEREAU ${ }^{1}$}

${ }^{1}$ EA 2114 / Psychologie des âges de la vie et adaptation, UFR Arts et Sciences Humaines, université de Tours, 3 rue des Tanneurs, 37000 Tours

${ }^{2}$ Service d'Onco-Hématologie pédiatrique, CHU Clocheville, boulevard Béranger, 37000 Tours

${ }^{3}$ Psychologue, service d'oncologie ;

Hôpital Nord-Franche-Comté ; 1 rue Henri Becquerel, 25200 Montbéliard

* Auteur correspondant : Philippe Colombat

EA 2114 / Psychologie des âges de la vie et adaptation, UFR Arts et Sciences Humaines, université de Tours, 3 rue des Tanneurs, 37000 Tours

mail : philippe.colombat@univ-tours.fr 


\section{Mieux manager pour mieux soigner}

\section{A better management for a better care}

\section{Résumé}

L'épuisement professionnel des soignants est particulièrement fréquent en cancérologie. Ses causes en sont plurifactorielles, car relevant quasiment toujours de l'association de facteurs personnels et professionnels. Ceux liés au travail peuvent être séparés en cinq catégories : le type de travail, la charge de travail, les conflits interpersonnels, les facteurs organisationnels et les facteurs managériaux. La qualité de vie au travail est un concept plus récent qui s'inscrit dans le champ de la psychologie positive. La démarche participative est un modèle organisationnel qui reposait initialement sur 4 composantes : la formation interne, les staffs pluriprofessionnels, le soutien aux équipes et la démarche projet. Récemment nous avons rajouté une cinquième composante devant le constat de la nécessité d'espaces d'échanges entre les médecins et les cadres de santé des services pour que le modèle marche. Ce modèle est un critère prioritaire HAS d'accréditation des établissements depuis la V 2010 pour la prise en charge des patients en soins palliatifs dans tous les services de soins. Dans la dernière partie de cet article nous verrons l'impact des facteurs managériaux et organisationnels mais également du modèle organisationnel de la démarche participative sur la qualité de vie au travail des soignants mais également sur la qualité des soins offerts aux patients et à leurs proches.

Mots-clés : cancérologie ; qualité de vie au travail ; souffrance au travail ; management ; soins de support

\section{Abstract}

The frequency of personal exhaustion in particularly high in oncology. In most cases its causes are related to an association of personal and professional problems. Factors connected to the work can be separated in five categories: type of work, work overload, interpersonal conflicts, organizational and managerial factors. The quality of work life is a more recent concept and joins in the field of the positive psychology. The participative approach is an organizational model which rested initially on 4 components: internal formation, team support meetings, pluriprofessionnal staffs and project approach. More recently we added a fifth component because we noticed that the model could work only if there were meetings between the doctors and the head nurses of the services. This model is a priority criterion of HAS accreditation of establishments since 2010 for the management of patients in palliative care in all services. In the last part of this article, we shall see the impact of the managerial and organizational factors but also of the organizational model of the 
participative approach on the quality of work life of caregivers but also on the quality of care offered to the patients and to their close friends.

Keywords: oncology; quality of work life; work suffering; management; supportive care

L'épuisement professionnel et plus récemment la qualité de vie au travail des soignants (QVT-S) est devenue une préoccupation du travail, de l'Agence Nationale d'Amélioration des Conditions de Travail (ANACT) et encore plus récemment de la Haute Autorité de Santé (HAS) et du ministère de la santé. Cet intérêt pour la qualité de vie au travail des soignants a conduit à la mise en place récente de plusieurs programmes tels que le programme PACTE sur l'amélioration du travail en équipe par l'HAS [1] et le programme CLUSTER [2] sur l'amélioration de la qualité de vie au travail par l'accompagnement d'établissements de santé par l'ANACT. La santé au travail est devenue un critère HAS d'accréditation des établissements dans la V 2014 [3]. Fin 2016 le ministère de la santé a par ailleurs lancé la stratégie nationale d'amélioration de la qualité de vie au travail avec trois composantes, la mission nationale, la médiation et l'observatoire national de la qualité de vie au travail des personnels de santé [4].

La cancérologie est l'une des spécialités où la fréquence de l'épuisement professionnel est la plus importante. Cependant ces taux sont très variables d'un service à l'autre.

Après avoir évoqué la fréquence et les facteurs déterminants de l'épuisement professionnel des soignants, nous détaillerons le modèle organisationnel innovant de la démarche participative et de son impact sur la qualité de vie au travail et sur la qualité des soins.

\section{L'épuisement professionnel des soignants en cancérologie}

Le burn-out ou syndrome d'épuisement professionnel des soignants a fait l'objet de différentes définitions. Communément il est néanmoins identifié comme un état transitoire lié à des circonstances professionnelles défavorables, mais n'est pas une maladie.

En 1974, H. Freudenberger a été le premier à décrire « un état causé par l'utilisation excessive de son énergie qui provoque le sentiment d'être épuisé et d'avoir échoué » [5]. D. Bédard et A. Duquette le définissent comme une « expérience psychologique négative vécue 
par un individu, liée au stress émotionnel et chronique causé par un travail ayant pour but d'aider les gens », définition applicable au monde soignant [6].

Les études montrent une fréquence de burn-out variable en fonction des spécialités et des professions : entre 10 et $40 \%$ pour les soignants non-médecins et entre 20 et $70 \%$ pour les médecins. La fréquence de l'épuisement professionnel est particulièrement élevée dans les services de cancérologie, toutes les études montrant des pourcentages de 20 et $40 \%$ pour les infirmières et les aides-soignantes [7], de 40 à $60 \%$ pour les oncologues [8] et de 50 à $70 \%$ pour les internes en oncologie [9].

\section{Le syndrome d'épuisement professionnel des soignants se définit par trois dimensions,} chacune étant scientifiquement évaluable :

- L'épuisement émotionnel

- La déshumanisation de la relation à l'autre

- La perte de sens et de l'accomplissement de soi au travail

Ces trois dimensions sont indépendantes, chacune de ces composantes étant vécues à des degrés divers, le profil variant en fonction de la profession [7].

La qualité de vie au travail est un concept plus récent relevant de la psychologie positive. Pour la HAS, " la qualité de vie au travail a comme objectif de concilier les modalités de l'amélioration des conditions de travail et de vie pour les salariés et la performance collective de l'entreprise. Elle fait valoir que la perception qu'ont les salariés de la qualité de vie au travail dépend de leur capacité à s'exprimer et à agir sur le contenu de leur travail. Elle met en avant le rôle des salariés et de leurs représentants en tant qu'acteurs de la construction des solutions proposées, notamment organisationnelles, aux côtés des directions, de l'encadrement et des experts. " [10].

Qu'il s'agisse de l'épuisement professionnel ou de la qualité de vie au travail, il existe de nombreuses échelles validées permettant de les explorer (www.afsos.org). L'outil le plus utilisé pour dépister le syndrome d'épuisement professionnel des soignants (MBI test) a été développé par C. Maslach et S. E. Jackson en 1981 [11]. Ce questionnaire de 22 items investigue les 3 dimensions du syndrome d'épuisement professionnel des soignants.

\section{Les causes de développement du syndrome d'épuisement professionnel des soignants}




\section{Elles sont toujours plurifactorielles.}

Le soignant présentant un épuisement professionnel le perçoit rarement : c'est donc habituellement son entourage et ses collègues qui s'en rendent compte par son changement de comportement (troubles de la concentration, irritabilité, repli sur soi, troubles de l'appétit et du sommeil, absentéisme)

\section{$1^{\circ}$ Rechercher une cause personnelle}

Le syndrome d'épuisement professionnel des soignants est quasiment toujours lié à l'association de causes personnelles et professionnelles. En dehors de facteurs favorisants tels les traits de personnalité (pessimisme, anxiété, difficulté d’adaptation, perfectionnisme), un idéal soignant élevé, la difficulté de trouver la " juste distance », il faut également rechercher un événement récent dans la vie personnelle pouvant être à l'origine d'une décompensation : retour de grossesse, difficultés financières et/ou de couple, séparation, deuil récent ou deuil ancien non élaboré.

\section{$\underline{2^{\circ} \text { Analyser les différentes causes professionnelles }}$}

L'association Francophone des Soins Oncologiques de Support (AFSOS) [12-13] et l'ANACT ont proposé des modèles explicatifs de l'épuisement professionnel relativement proches [14]. L'AFSOS a catégorisé cinq causes de stress professionnel [12-13] :

Les facteurs liés à la discipline et à la profession :

Être aide-soignant, infirmière, médecin, travailler en pédiatrie, gériatrie, chirurgie ou oncologie ne procure pas les mêmes types de stress.

On décrit une plus forte prévalence dans les services accueillant des patients atteints de maladie grave, voire mortelle, où les sollicitations émotionnelles sont les plus prégnantes (patients jeunes ou au contraire très âgés, confrontation répétée avec la souffrance et la mort, conflits éthiques non résolus), comme en cancérologie. Il en est de même pour les services à fort risque de gravité des erreurs potentielles comme la réanimation ou des spécialités à pratique très répétitive (urgences, anesthésie).

La charge de travail :

Charge de travail excessive, ratio de personnel insuffisant, manque de travail en binôme et/ou en équipe. S'il apparaît qu'une charge de travail trop excessive joue un rôle, plusieurs études ont montré, tant au niveau médical que paramédical, que la durée de travail ne jouait pas de rôle, la qualité du travail étant plus importante que la durée. 
Les conflits interpersonnels

Au sein de l'équipe, en particulier ceux liés aux personnalités pathologiques.

L'organisation du travail

En insistant particulièrement sur l'ambiguïté des rôles, l'interruption des tâches et l'absence de justice dans la gestion du planning.

Le management

Sur lequel nous reviendrons, en soulignant dès à présent que si un bon manager a recours à 4 types majeurs de management (directif, persuasif, participatif et délégatif) selon les situations et les circonstances (management circonstanciel), l'utilisation exclusive des managements directif et persuasif provoque, elle, de la souffrance au niveau des équipes. Les conséquences de la qualité du management sont multiples avec un retentissement par exemple sur la fréquence des conflits personnels et sur la qualité de l'organisation. Le manager joue également un rôle essentiel dans la gestion des changements imposés par les circulaires et les lois successives en permettant de réfléchir en groupes de travail à leur mise en place et à leurs conséquences.

Si nous ne pouvons développer dans le détail la prévention, le dépistage et la prise en charge du syndrome d'épuisement professionnel des soignants (voir référentiel AFSOS/SFPO [13]), la connaissance des causes qui en sont à l'origine permet de définir des mesures de prévention combinant une prévention individuelle (prendre soin de soi pour prendre soin des autres: formations à la communication, à la relation, aux techniques de relaxation et pratique de ces techniques de développement des ressources personnelles) et une prévention collective au niveau des services par la mise en place d'un management et d'une organisation adaptés.

\section{La démarche participative}

\section{Historique [15]}

L'élaboration du concept de la « Démarche Palliative » (1992-1998).

Dans les années 1990, le Groupe de Réflexion sur l'Accompagnement et les Soins palliatifs en Hématologie (GRASPH) constatant la fréquence de la souffrance des soignants en Hématologie, élabore un modèle de management d'équipe qui permettrait de mieux prendre en charge notamment les malades les plus en souffrance et les plus complexes dans les services d'hématologie que sont les patients en phase palliative. C'est ainsi, tout 
naturellement que ce modèle de management / Démarche Participative est dénommé « Démarche Palliative ».

Le temps des textes législatifs (1998-2008).

2002 : $1^{\text {re }}$ circulaire du ministère de la Santé prônant la mise en place de la démarche palliative dans les services de soins comme modèle de prise en charge des patients en soins palliatifs.

2004 (juin) : Circulaire ministérielle «Guide de la mise en place de la démarche palliative en établissement » décrivant le modèle de la démarche palliative.

2008 : Circulaire du 26 mars relative à l'organisation des soins palliatifs.

2008 I'HAS entérine le caractère obligatoire de la circulaire en faisant de la démarche palliative un des cinq critères prioritaires d'accréditation pour les établissements de court, moyen et long séjour sous forme de la mesure 13a V 2010.

La démonstration scientifique

En 2004, il devint évident qu'il était nécessaire de vérifier ce que nous affirmions depuis presque 15 ans à savoir qu'une démarche participative d'équipe améliorait la qualité de vie au travail des soignants. Nous avons alors mis en place dans le monde de la santé mais aussi de l'entreprise des études analysant l'impact des facteurs organisationnels et managériaux sur la qualité de vie au travail et plus spécifiquement les effets de la démarche participative sur la qualité de vie au travail des soignants et sur la qualité des soins.

Objectifs actuels

Ils sont doubles :

- Proposer de généraliser le modèle à la prise en charge des patients porteurs de maladie chronique.

- Former les managers de la santé que sont les médecins, les cadres de santé et les directeurs d'établissement à la démarche participative. C'est pourquoi l'AFSOS a actuellement une structure de formation qui travaille avec l'université de Tours et d'autres structures de formation sur des programmes d'un ou deux jours et plus récemment sur une sensibilisation en ligne sur internet.

\section{$\underline{2^{\circ} \text { Le modèle organisationnel de la démarche participative [15] }}$}


Ce modèle organisationnel avait initialement 4 composantes :

- Les formations internes aux équipes. La formation continue consiste habituellement, pour les soignants à participer soit à des formations organisées au sein de l'établissement et regroupées dans le plan de formation, soit à des formations extérieures ou à des congrès. En revanche, les formations au sein des services impliquant toutes les catégories socioprofessionnelles sont plus inhabituelles. En dehors des apports théoriques, elles permettent de fédérer les équipes, de mieux se connaître et d'échanger sur le vécu de la thématique au sein du service, la réussite de ces temps d'échanges étant très liée aux talents de conduite de réunion du formateur [16-17].

- Les staffs de débriefing, à ne pas confondre avec les réunions de supervision, sont connus depuis de nombreuses années dans certaines spécialités notamment la réanimation, les urgences... À organiser dans les 72 heures suivant la situation, ils sont animés par un psychologue extérieur au service. Non obligatoires, ils offrent aux soignants un espace de parole par rapport à une situation ayant mis l'équipe dans la difficulté [18-19].

- Les staffs pluriprofessionnels, sans aucun doute la composante la plus importante pour la qualité de prise en charge des patients et de leurs proches. L'AFSOS a déjà beaucoup travaillé sur le déroulé et le contenu de ces staffs et des recommandations sont en cours de rédaction. En 2016, en collaboration avec l'HAS, la rédaction d'une grille d'évaluation des staffs pluriprofessionnels a été rédigée. II existe par ailleurs des documents de références (Les staffs d'une équipe médico-soignante, HAS [20]). Nous insistons notamment sur l'ordre de prise de parole en recommandant que les aides-soignantes et les infirmières parlent en premier et les médecins en dernier et nous préconisons la technique des trois tours de table, le premier pour cerner les besoins du patient et de ses proches, le deuxième pour proposer des schémas de prise en charge et le dernier pour valider la proposition qui fait consensus. Ces réunions, au cours desquelles est abordées la prise en charge des patients permettent de réinterroger les prises en charge et de proposer des améliorations en identifiant, voire en modifiant les normes, les valeurs et les modèles mentaux sous-jacents au travail clinique, engageant ainsi les professionnels dans un apprentissage en continu (double-loop learning). Les débriefings et réunions cliniques améliorent en moyenne de 20 à $25 \%$ la performance des équipes [21].

- La démarche projet est largement utilisée dans tous les milieux socio-professionnels avec une méthodologie bien réglée. Dans les services de soins, elle peut revêtir une 
approche globale sous la forme d'un projet de service ou une approche plus ciblée sous la forme de groupes de travail sur des problématiques de terrain soulevées par les patients ou les soignants. La pluriprofessionnalité des groupes de travail et la qualité des échanges et de la communication sont les garants de la réussite de la démarche. Là encore l'HAS a émis des recommandations sur la démarche de projet de service (outil pour l'amélioration des pratiques, HAS [22]).

Le modèle de la démarche participative est un modèle de travail en équipe qui vise à :

- Répondre aux difficultés de terrain propres aux services et aux équipes par une recherche d'adéquation entre le management et le contexte dans lequel il intervient.

- Générer des effets de groupe positifs favorisant le développement d'une intelligence collective appuyée sur des qualités de respect, reconnaissance de soi et de l'autre, de tolérance, de collaboration, de confiance et d'humilité.

- Impliquer les soignants comme acteurs/partenaires.

En 2008 nous avons ajouté une cinquième composante qui nous semble également indispensable : la création d'un espace d'échanges réguliers entre les managers du service que sont TOUS les médecins et les cadres pour les prises de décision organisationnelles. En effet, nous nous sommes rendu compte que seul ce type de réunion permettait l'acquisition par les différents managers de valeurs et de pratiques managériales communes.

\section{Focus sur management et qualité de vie au travail.}

La qualité de vie au travail (qualité de vie au travail) s'inscrit dans le champ de la psychologie positive. Elle est communément définie comme le niveau d'efficacité personnelle perçue par les personnes dans les quatre domaines de leur activité professionnelle: physique, psychologique, social et culturel [23].

Le manque de reconnaissance est le facteur reconnu comme altérant le plus la qualité de vie au travail (qualité de vie au travail) dans la plupart des études [24-25]. Concernant les facteurs organisationnels et managériaux, le management transformationnel [26] (un manager exemplaire pratiquant un management individualisé et qui fait confiance aux soignants), le soutien à l'autonomie [27], la justice organisationnelle [28] et le soutien organisationnel perçu [29] apparaissent en revanche dans la littérature scientifique comme les facteurs les plus déterminants de la qualité de vie au travail. 
Facteurs organisationnels et managériaux et qualité de vie au travail des soignants Données de la littérature

Les données de la littérature montrent que les facteurs organisationnels et managériaux peuvent avoir des effets significatifs sur l'état de santé, les attitudes et les comportements des soignants [30-31]. Par exemple, dans l'étude de Verhaeghe et al. [32] le degré d'autonomie dans le travail apparaît inversement corrélé au niveau de stress dans l'activité professionnelle. Estryn-Behar, et al. [33] ont relevé que l'organisation du travail interdisciplinaire adoptée par les équipes de soins palliatifs permettait de limiter le développement de l'épuisement professionnel chez les soignants. Toujours en dehors du domaine de l'Oncologie, Gunnarsdottir et al [34] utilisant l'échelle Nursing Work Index Revised (NWI-R), échelle infirmière d'auto-évaluation de la qualité de prise en charge, et le $\mathrm{MBI}$, ont démontré des corrélations entre la perception de soutien des managers, l'adéquation des réunions et la qualité des relations entre infirmières et médecins d'une part, et la satisfaction au travail, l'épuisement émotionnel et la qualité des soins évalués par les infirmières d'autre part. Plus spécifiquement dans le champ de l'oncologie, Cummings et al [35] ont étudié la relation entre le management et la satisfaction au travail de 515 infirmières travaillant dans des services d'oncologie au Canada : le type de management, notamment la qualité de la relation entre les médecins et les infirmières, influence significativement et positivement les opportunités pour améliorer les réunions, l'autonomie des infirmières, leur participation aux décisions d'organisation, la mise en place d'innovations, la gestion des conflits, aboutissant ainsi à une amélioration de leur satisfaction au travail. Dans la littérature, les principales publications dans ce domaine sont relatives au modèle des « Hôpitaux magnétiques » mis en place dans certains hôpitaux canadiens et américains à la fin des années 90 pour attirer et retenir les personnels soignants (le modèle du " lean management », transfert du monde industriel et actuellement développé aux États-Unis aboutissant à des résultats très controversés [36]). Ce modèle repose notamment sur un soutien à l'autonomie clinique, un soutien organisationnel du cadre de santé, une politique de formation et une bonne collaboration entre médecins et infirmières, un contrôle de la qualité des soins et la transmission d'une culture de soins centrée sur le patient. Ces travaux ont montré que les personnels soignants travaillant dans ces hôpitaux ont, dans les études de comparaison avec des hôpitaux 
classiques, une meilleure qualité de vie au travail de leurs personnels soignants, se traduisant par une diminution du turnover et de l'absentéisme, un meilleur engagement au travail, et une meilleure évaluation de la qualité des soins prodigués [37].

\section{Démarche participative et qualité de vie au travail des soignants}

En 2004 notre groupe a obtenu un financement «Programme Hospitalier de Recherche Clinique ". L'étude visait à démontrer une relation entre un management participatif et la qualité de vie au travail (qualité de vie au travail) des soignants de services d'oncohématologie du Cancéropôle Grand Ouest [17].

Cinq cent soixante-quatorze infirmières et aides-soignantes ont été incluses dans cette étude dont les résultats montrent des relations négatives entre d'une part les manques de reconnaissance au travail, de formation, de collaboration au sein de l'équipe et l'absence de staffs pluriprofessionnels et d'autre part la qualité de vie au travail. Pour ces mêmes facteurs, des corrélations positives ont été observées pour le stress perçu (avec comme facteurs supplémentaires le nombre de lits par service et le nombre de décès par mois) et des corrélations négatives pour le soutien social (avec comme facteurs supplémentaires le temps de transmission et la présence de bénévoles d'accompagnement) (voir tableau 1).

En 2009 un deuxième programme hospitalier de recherche clinique a retrouvé les mêmes résultats auprès d'une population de 323 soignants de même spécialité. Cette deuxième étude en cours de publication s'est attachée à étudier l'impact des composantes du management du cadre de santé. II apparaît :

$\checkmark$ que le leadership transformationnel (management responsabilisant les agents) entraîne une meilleure qualité de vie au travail et un meilleur engagement dans le travail des soignants grâce à un renforcement des sentiments de justice organisationnelle (voir la Figure 1 ci-dessous) [38].

$\checkmark$ que la justice procédurale (perception de justice dans les procédures mises en place) et le soutien à l'autonomie du cadre de santé améliorent la satisfaction des besoins psychologiques (i.e., les salariés se sentent plus autonomes et plus compétents, et entretiennent de meilleures relations interpersonnelles avec leurs collègues) et le soutien organisationnel perçu par les soignants, entraînant ensuite une meilleure satisfaction au travail, un renforcement de l'identification au service et une amélioration de la performance au travail [39]. 
Très récemment, dans une étude encore en cours de publication, nous avons étudié l'impact de la Démarche Participative et de ses composants (formation interne, staffs pluriprofessionnels, soutien aux équipes, démarche projet) sur la Qualité de Vie au Travail des soignants en oncopédiatrie ainsi que leur impact sur la qualité de prise en charge des enfants. A notre connaissance, il s'agit de la première étude mondiale ayant étudié sur un grand échantillon la Qualité de Vie au Travail des soignants et la qualité de prise en charge des enfants en oncopédiatrie, ainsi que les déterminants managériaux et organisationnels de celles-ci. Les questionnaires que nous avons utilisés sont l'échelle d'Elizur et Shye [15] pour la qualité de vie au travail, l'Utrecht Work Engagement Scale (UWES) [40] pour l'engagement au travail, une échelle de Likert graduée de 1 à 5 pour l'indice de satisfaction au travail et la qualité des soins perçue par l'équipe.

Cinq cent dix soignants de 25 des 30 centres d'oncologie pédiatrique français ont participé à cette étude. Les résultats ont objectivé une relation significative entre la Démarche Participative et la Qualité de Vie au Travail des soignants $\left(p<10^{-5}\right)$ tant au niveau santé globale $\left(p<10^{-5}\right)$ qu'au niveau de la Qualité de Vie au Travail psychologique $\left(p<10^{-5}\right)$, sociale $\left(p<10^{-4}\right)$, culturelle $\left(p<10^{-5}\right)$ et physique $(p<0.05)$. Les quatre composants analysés séparément sont également très fortement corrélés à la qualité de vie au travail : la formation $(p<0.001)$, les staffs $\left(p<10^{-4}\right)$, la démarche projet $\left(p<10^{-4}\right)$ et le soutien aux équipes $(p<0.01)$.

La Démarche Participative influence également de manière positive l'engagement au travail des soignants $\left(p<10^{-3}\right)$, la satisfaction au travail des soignants $\left(p<10^{-3}\right)$; la démarche projet et les staffs apparaissent comme les composantes de la Démarche Participative influençant le plus les marqueurs d'impact de la Qualité de Vie au Travail étudiés, notamment concernant la satisfaction des soignants vis-à-vis de la qualité des soins.

\section{Facteurs organisationnels et managériaux et qualité des soins}

\section{Données de la littérature}

L'impact sur la qualité des soins n'a été démontré que dans peu d'études. Vahey et al [41] ont rapporté une étude simultanée auprès de 820 infirmières et de 621 patients de 40 unités de soins américaines. Les patients pris en charge dans les unités décrites par les infirmières comme ayant un effectif adéquat, un bon soutien de l'administration et de bonnes relations entre médecins et infirmiers ont été plus du double à déclarer une haute satisfaction des soins par rapport à ceux des autres unités. Par ailleurs les 
infirmiers de ces unités ayant une bonne organisation et des relations internes satisfaisantes présentaient significativement moins de burn-out : ainsi l'amélioration de l'environnement professionnel peut réduire simultanément le burn-out et le turnover tout en augmentant la satisfaction des patients.

Plus récemment Van Beek et al [42] décrivent une relation entre une culture de clan des équipes, caractérisées par une forte cohésion, une bonne participation et le sens de l'intérêt commun et la qualité des soins perçue par les soignants et observée par les chercheurs.

Aiken, Sermeus, Van den Heede, et al. [43] ont analysé les effets des conditions de travail des personnels soignants (e.g., nombre de patients par infirmier, participation des personnels soignants à la prise de décision) sur la qualité des soins dans douze pays européens et aux États-Unis. Leurs résultats ont démontré que de bonnes conditions de travail étaient associées à une meilleure qualité des soins évaluée aussi bien par les soignants que les patients.

Dans le domaine de la cancérologie, Friese et al. [44] ont analysé les effets des caractéristiques de l'environnement professionnel des personnels soignants (e.g., relations entre les médecins et les infirmiers, pratiques managériales) sur la qualité des soins délivrée aux patients atteints de cancer. Ces chercheurs ont montré que les complications étaient plus nombreuses et la mortalité des patients plus élevée dans les établissements de santé proposant les moins bonnes conditions de travail. Friese et Manojlovich [45] ont quant à eux analysé les perceptions des infirmiers de leurs relations avec les médecins dans le contexte de l'oncologie ambulatoire. Ils ont également demandé aux infirmiers d'évaluer la qualité des soins délivrée aux patients. Les résultats ont démontré que de bonnes relations entre les médecins et les infirmiers étaient positivement associées à la qualité des soins. Aussi, comme nous l'avons déjà mentionné, il semble possible d'améliorer la sécurité et la qualité des soins dans les services de cancérologie en renforçant la communication et la collaboration entre les différents personnels soignants.

Enfin, dans le modèle des « hôpitaux magnétiques », West E et al. [46] montrent en 2001 une amélioration de la satisfaction des patients. Plusieurs auteurs montrent que le travail en équipe, outre son impact sur la sécurité des soins, est un facteur de qualité de prise en charge du patient et un moyen de répondre de manière collaborative et interdisciplinaire aux besoins des patients [47-49] 


\section{$\underline{\text { Impact de la démarche participative sur la qualité des soins }}$}

Dans l'étude précédemment citée concernant l'oncologie pédiatrique, nous avons également testé l'impact du modèle organisationnel de la démarche participative sur la satisfaction de la qualité des soins évaluée par les soignants et la qualité de prise en charge des enfants via le questionnaire SAPHORA-MCO le plus utilisé en France [50], (le questionnaire étant rempli par les enfants âgés de 12 ans et plus et par les parents pour les enfants de moins de 12 ans).

Il existe une relation très significative entre la démarche participative et la qualité des soins évaluée par les soignants $\left(p<10^{-5}\right)$. Pour l'évaluation de la qualité de prise en charge des enfants 440 questionnaires au total (142 questionnaires remplis par les enfants et 298 questionnaires remplis par les parents) ont pu être collectés. Si on constate une bonne corrélation significative et positive entre la qualité de prise en charge évaluée par les soignants et par les parents $(p<0,01)$, on retrouve à l'inverse une corrélation non significative au niveau des centres, entre l'évaluation de la qualité de prise en charge par les parents et celle faite par les enfants $(p=0,33)$, pouvant peut-être s'expliquer en partie par le fait que le questionnaire SAPHORA-MCO est un outil conçu et utilisé en médecine adulte.

Par ailleurs, il a été mis en évidence que certaines composantes de la Démarche Participative influencent positivement certains aspects de la qualité de prise en charge des enfants : les staffs pluriprofessionnels sont ainsi corrélés positivement avec la satisfaction des enfants et des parents vis-à-vis de l'attitude des soignants $(p<0.01)$ et la composante soutien aux équipes a une influence positive sur la qualité de la communication avec les personnels de santé $(p<0.001)$.

\section{Corrélation entre la démarche participative et facteurs organisationnels et managériaux influençant la qualité de vie au travail des soignants}

II nous est paru intéressant de voir s'il existait une corrélation entre notre modèle de la démarche participative et les facteurs organisationnels et managériaux qui apparaissent essentiels dans la qualité de vie au travail des soignants.

À partir des données de notre récente étude en oncopédiatrie, nous avons ainsi pu observer des corrélations très importantes et positives entre notre modèle d'une part et les facteurs 
managériaux que sont le leadership transformationnel $\left(10^{-12}\right)$ et le soutien à l'autonomie (10$\left.{ }^{12}\right)$, les facteurs organisationnels que sont la justice organisationnelle $\left(10^{-20}\right)$ et le soutien organisationnel perçu $\left(10^{-21}\right)$ d'autre part. On retiendra également que chaque composante de la démarche participative est corrélée positivement avec ces facteurs organisationnels et managériaux, les composantes démarche projet et formation interne apparaissant les facteurs les plus fortement corrélés (voir tableau 2).

\section{Conclusion}

Nous avons vu que l'épuisement professionnel des soignants était particulièrement fréquent en cancérologie et que certaines de ses causes sont désormais bien connues. S'il est difficile d'impacter les causes liées à la discipline et à la profession, il apparaît essentiel, à partir des études que nous avons menées, d'agir, en dehors de la charge de travail, sur le management de proximité et de repenser le fonctionnement de la gouvernance.

La formation des managers, qu'il s'agisse des médecins, des cadres de santé ou des directeurs d'établissement apparaît donc un enjeu majeur pour demain, le fonctionnement des services et d'établissements devant impliquer tous les acteurs concernés (dont les patients) sur un modèle de bas en haut, de type participatif, et non plus de haut en bas de type directif.

\section{Remerciements}

Fondation de France; Association CANCEN; Plateforme Inca de qualité de vie au travail ; Société Française des Cancers de l'enfant

\section{Bibliographie}

1. https://www.has-sante.fr/portail/jcms/c_1733417/fr/experimentation-pacte

2. https://www.anact.fr/des-clusters-qualite-de-vie-au-travail-qvt-pour-les-etablissements-desante

3. https://www.has-sante.fr/portail/jcms/c_1772032/fr/guide-methodologique-v2014

4. https://solidarites-sante.gouv.fr/IMG/pdf/strategie_qvt_05122016.pdf

5. Freudenberger HJ. Staff burn-out. J Social Issues 1974; 30: 159-165

6. Bedard D ; Duquette A. L'épuisement professionnel, un concept à préciser - L'infirmière du Québec Septembre/octobre 1998, p 18-23 
7. Lissandre S., Abbey-Huguenin H., Bonnin-Scaon S., Arsene O., Colombat P.Facteurs associés au burn-out chez les soignants en oncohématologie. Oncologie 2008 ; 10 :L 116-124

8. Ramirez, A. J., Graham, J., Richards, M.A., et al. (1995). Burnout and psychiatric disorder among cancer clinicians. Br J Cancer, 71(6), 1263-1269.

9. Blanchard P, Rodrigues $\mathrm{M}$, Colombat P. On the prevalence and causes of oncologist burn-out. J Clin Oncol. $2012 ; 30(24): 3029-30$

10. https://www.has-sante.fr/portail/jcms/c_990756/fr/qualite-de-vie-au-travail

11. Maslach, C.; Jackson, S.E. (1981). "The measurement of experienced burn-out". Journal of Occupational Behavior. 2: 99-113.

12. Colombat $P$ (sous la direction de) Qualité de vie au travail et management participatif, Edition Lamarre (Paris) 2012

13. https://afsos.org/referentiels-recommandations

14. https://www.anact.fr/burnout-un-guide-pour-prevenir-le-syndrome-depuisement-professionnel

15. Colombat Ph, Bauchetet C, Fouquereau E, Gillet N, Kanitzer C. La démarche participative dans les soins. Gestions Hospitalières $2014 ; 534: 151-157$

16. Bauchetet $C$, Altmeyer A, Barruel $F$ et al. La démarche participative : un concept dynamique. Objectif Soins 2011; 198: 1-6

17. Meyer EM ${ }^{1}$, Zapatka S, Brienza RS.The development of professional identity and the formation of teams in the Veterans Affairs Connecticut Healthcare System's Center of Excellence in Primary Care Education Program (CoEPCE). Acad Med 2015; 90: 802-9

18. Hanna R, Rosanna M. Debriefing after a crisis? Nursing manag 2007; 39-47

19. Wrightington, Wigan and Leigh NHS Foundation Trust. Supporting staff wellbeing with stress management, mindfulness and trauma-debriefing. https://improvement.nhs.uk/documents

20. https://www.has-sante.fr/portail/jcms/c_2807236/fr/staffs-d-une-equipe-medico-soignantegroupes-d-analyse-de-pratiques-gap

21. Huzzard, T., Welp, A., \& Kolbe, M. (2016). Teamwork in healthcare organizations. In A. Örtenblad, C. Abrahamson Löfström, \& R. Sheaff (Eds.), Management Innovations for Health Care Organizations: Adopt, Abandon or Adapt? (Vol. 18, pp. 359-377). Routledge. https://www.researchgate.net/profile/Jan_Schmutz/ publication /291342147_Teamwork_in_Healthcare_Organizations/links/56a0db5708aee4d26ad8b613 /Teamwork-in-Healthcare-Organizations.pdf

22. https://www.has-sante.fr/portail/upload/docs/application/pdf/201610/outil_12_guide_elaboration_projet_equipe.pdf

23. Elizur, D, Shye, S. Quality of work and its relation to quality of life. Applied Psychology : An International Review 1990;39, (3), 275-291.

24. Pronost, A.-M., Le Gouge, A., Leboul, D., Gardembas-Pain, M., Berthou, C., Giraudeau, B., ... Colombat, P. Relationships between the characteristics of oncohematology services providing palliative care and the sociodemographic characteristics of caregivers using health indicators : social support, perceived stress, coping strategies, and quality of work life. Supportive Care in Cancer; 2012; 20(3): 607-614

25. Dejours C. Psychanalyse et psychodynamique du travail : ambiguïtés de la reconnaissance. In $A$ Caillé. La quête de reconnaissance. Edition La découverte, 2007, p 66

26. Givens JR. Transformational leadership: the impact on organizational and personal outcomes. Emerging leadership journeys 2008; 1:4-24

27. Gillet N, Gagne M, Sauvagere $S$ et Fouquereau M. The role of supervisor autonomy support, organizational support and autonomous and controlled motivation in predicting 
employees'satisfaction and turnover intentions. European Journal of Work and Organizational Psychology 2013; 44 : 450-460

28. Liljegren $M$, Ekberg $K$. The associations between perceived distributive, procedural and interactional organizational justice, self'rated health and burn-out. Work 2009; 33 : 43-51

29. Brunault P, Fouquereau E, Colombat P, Gillet N, El-Hage W, Camus V et Gaillard P. Do transactive and participative teamwork improve nurses'quality of work life? Western Journal of Nursing Research 2014; 36: 329-45

30. Eelen, S., Bauwens, S., Baillon, C., Distelmans, W., Jacobs, E., Verzelen, A. (2014). The prevalence of burn-out among professionals; oncologists are at risk of developing burn-out. Psychooncology, 23, 1415-1422

31. Jaspaerse, M., Herst, P., Dungey, G. (2014). Evaluating stress, burn-out and job satisfaction in New Zealand radiation oncology department. Eur J Cancer Care, 23, 83-88

32. Verhaeghe, R., Mak, R., Van Maele, G., Kornitzer, M., De Baker, G. Job stress among middle-aged health care workers and its relationship to sickness absence. Stress and Health; 2003; 19: 265-274.

33. Estryn-Behar, M., Lassauniere, J. M., Fry, C., de Bonnieres, A. L'interdisciplinarité diminue-t-elle la souffrance au travail ? Comparaison entre soignants de toutes spécialités (médecins et infirmiers) avec ceux travaillant en soins palliatifs, en onco-hématologie et en gériatrie. Médecine Palliative 2011; 11: 65-89

34. Gunnarsdóttir, S., Clarke, S. P., Rafferty, A. M., \& Nutbeam, D. (2009). Front-line management, staffing and nurse-doctor relationships as predictors of nurse and patient outcomes. A survey of Icelandic hospital nurses. International. Journal of Nursing Studies, 46(7), 920-927

35. Cummings, G. G., Olson, K., Hayduk, L., Bakker, D., Fitch, M., Green, E., ... Conlon, M. The relationship between nursing leadership and nurses' job satisfaction in Canadian oncology work environments. Journal of Nursing Management; 2008; 16(5): 508-518.

36. Rust, T. C. Dynamic Analysis of Healthcare Service Delivery: Application of Lean and Agile Concepts. Worcester Polytechnic Institute.2013; http://www.wpi.edu/Pubs/ETD/Available/ etd043013-140924/

37. Lundstrom, T, Pugliese, G., Bartley, J., Cox, J., Guither, C. (2002). Organizational and environmental factors that affect worker health and safety and patient outcomes. Am J Infect Control, 30, 93-106.

38. Gillet, N., Fouquereau, E., Bonnaud-Antignac, A., Mokounkolo, R., \& Colombat, P. The mediating role of organizational justice in the relationship between transformational leadership and nurses' quality of work life: A cross-sectional questionnaire survey. International Journal of Nursing Studies ; 2013 ; 50(10) : 1359-1367.

39. Gillet, N., Colombat, P., Michinov, E., Pronost, A.-M., \& Fouquereau, E. Procedural justice, supervisor autonomy support, work satisfaction, organizational identification, and job performance: The mediating role of need satisfaction and perceived organizational support. Journal of Advanced Nursing; 2013; 69: 2560-2571.

40. Zecca G, Györkös C, Becker J et al. Validation of the French Utrecht Work Engagement Scale and its relationship with personality traits and impulsivity. Eur Rev Appl Psychol 2015; 1: 19-28.

41. Vahey, D. C., Aiken, L. H., Sloane, D. M., Clarke, S. P., \& Vargas, D. (2004). Nurse Burnout and Patient Satisfaction, Medical Care, 42(Suppl), II57-II66 
42. Van Beek AP, Gerritsen DL. The relationship between organisational culture of nursing staff and quality of care for residents with dementia : questionnaire surveys and systematic observations in nursing homes. Int J Nurs studies 2010. 47: 274-82

43. Aiken, L. H., Sermeus, W., Van den Heede, K., et al. Patient Safety, Satisfaction, and Quality of Hospital Care: Cross Sectional Surveys of Nurses and Patients in 12 Countries in Europe and the United States. British Medical Journal; 2012; 344: 1-14.

44. Friese, C. R., Lake, E. T., Aiken, L. H., Silber, J. H., Sochalski, J. Hospital nurse environments and outcomes for surgical oncology patients. Health Serv Res; 2008; 43 : 1145-1163.

45. Friese, C. R., Manojlovich, M. (2012). Nurse-physician relationship in ambulatory oncology settings. J Nurs Scholarsh; 2012; $44:$ 268-275.

46. West E. Management matters: the link between hospital organisation and quality of patient care. Quality in Health Care, 2001; 10: 40-48

47. Baxter SK, Brumfitt SM. Benefits and losses: a qualitative study exploring healthcare staff perceptions of teamworking. Qual Saf Health Care 2008;17(2):127-30.

48. Neily J, Mills PD, Young-Xu Y et al. Association between implementation of a medical team training program and surgical mortality. JAMA 2010;304(15):1693-700

49. Zwarenstein $M$, Goldman J, Reeves S. Interprofessional collaboration: effects of practice-based interventions on professional practice and healthcare outcomes. Cochrane Database Syst Rev 2009;(3):CD000072.

50. Salomon L, Gasquet I, Mesbah M, Ravaud P. Construction of a scale measuring patient's opinion on quality. Int J Qual Health Care 1999; 6: 507-527 
Figure 1. Résultats obtenus par Gillet, Fouquereau, Bonnaud-Antignac, Mokounkolo et Colombat [38]

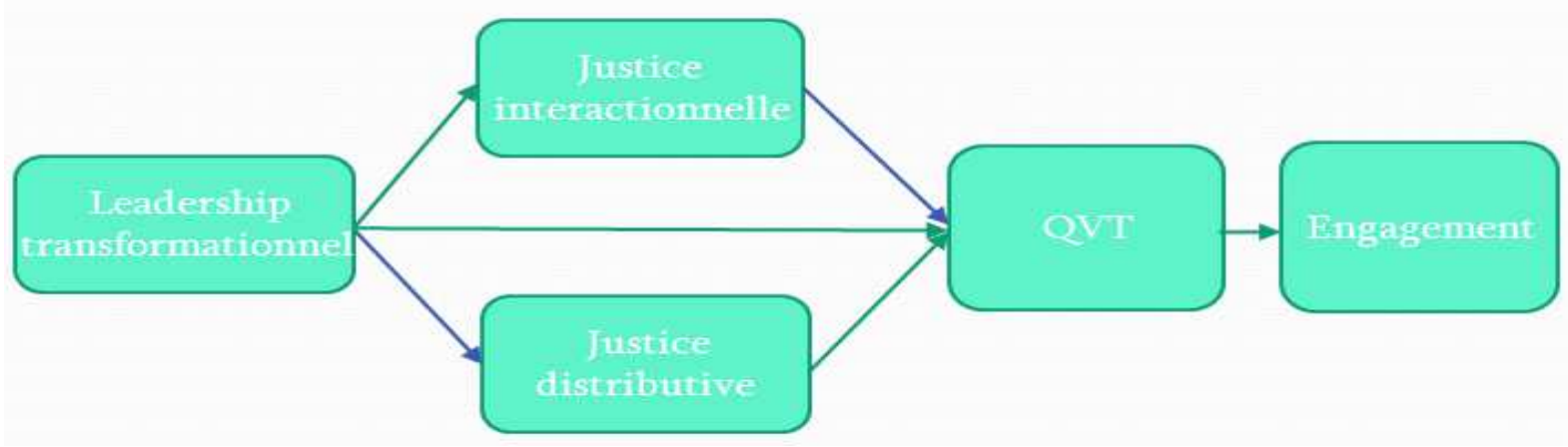


Tableau 1 : facteurs prédictifs de la QVT (Pronost et al ; 2012)

\begin{tabular}{|l|c|c|}
\hline \multicolumn{1}{|c|}{ Variables } & $\begin{array}{c}\text { Qualité de vie } \\
\text { au travail }\end{array}$ & $p$ \\
\hline Manque de reconnaissance & $-0,32$ & $<0,001$ \\
\hline Manque de temps & $-0,27$ & 0,001 \\
\hline Mauvaise prise en compte des besoins des patients & $-0,21$ & 0,002 \\
\hline Mauvaise prise en compte des besoins des proches & $-0,21$ & 0,001 \\
\hline Manque de formation & $-0,18$ & 0,003 \\
\hline Manque de collaboration & $-0,16$ & 0,008 \\
\hline Aucune réunion interdisciplinaire & $-0,15$ & 0,034 \\
\hline Manque de communication & $-0,12$ & 0,038 \\
\hline
\end{tabular}


Tableau 2 : corrélation entre DP et facteurs organisationnels et managériaux

\begin{tabular}{|c|c|c|c|c|c|c|c|c|c|c|}
\hline \multirow{2}{*}{ FACTEURS } & \multicolumn{10}{|c|}{ DEMARCHE PARTICIPATIVE } \\
\cline { 2 - 11 } & \multicolumn{2}{|c|}{ Globale } & \multicolumn{1}{|c|}{ Formation } & \multicolumn{2}{|c|}{ Staffs } & \multicolumn{2}{c|}{ Soutien } & \multicolumn{2}{|c|}{ Projet } \\
\cline { 2 - 11 } & $r$ & $p$ & $r$ & $p$ & $r$ & $p$ & $r$ & $p$ & $r$ & $p$ \\
\hline SOP & .414 & $<10^{-21}$ & .291 & $<10^{-10}$ & .233 & $<10^{-6}$ & .27 & $<10^{-8}$ & .308 & $<10^{-11}$ \\
\hline LT & .324 & $<10^{-12}$ & .282 & $<10^{-9}$ & .110 & $<.05$ & .21 & $<10^{-5}$ & .253 & $<10^{-7}$ \\
\hline JO & .408 & $<10^{-20}$ & .337 & $<10^{-13}$ & .237 & $<10^{-6}$ & .19 & $<10^{-4}$ & .317 & $<10^{-12}$ \\
\hline SA & .323 & $<10^{-12}$ & .285 & $<10^{-9}$ & .101 & $<.05$ & .21 & $<10^{-5}$ & .268 & $<10^{-8}$ \\
\hline
\end{tabular}

